\title{
Comparison of Dissolution Profiles and Serum Concentrations of Two Lamotrigine Tablet Formulations
}

\author{
Mladena Lalic, ${ }^{1}$ Ana Pilipovic, ${ }^{1}$ Svetlana Golocorbin-Kon, ${ }^{1}$ Ksenija Gebauer-Bukurov, ${ }^{2}$ \\ Ksenija Bozic, ${ }^{2}$ Momir Mikov ${ }^{3,4}$ and Jelena Cvejic ${ }^{1}$ \\ 1 Department of Pharmacy, Faculty of Medicine, University of Novi Sad, Novi Sad, Republic of Serbia \\ 2 Institute of Neurology, Clinical Centre of Vojvodina, University of Novi Sad, Novi Sad, Republic of Serbia \\ 3 School of Pharmacy, University of Otago, Dunedin, New Zealand \\ 4 Department of Pharmacology, Toxicology and Clinical Pharmacology, University of Novi Sad, Novi Sad, \\ Republic of Serbia
}

Background: Since 2005, the antiepileptic drug lamotrigine has been present in the market in various generic products, in addition to the original brand of Lamictal ${ }^{\circledR}$. The linear pharmacokinetics and wide therapeutic window of lamotrigine enable seizure-free patients to easily switch from brand to generic antiepileptic drugs.

Objective: The aim of this study was to investigate the extent of variations in lamotrigine serum concentrations between two immediate-release tablet formulations. Data were compared with in vitro difference and similarity tests on dissolution profiles of the two formulations.

Methods: Dissolution characteristics of formulations A (reference) and B (test) were evaluated at three points spanning the physiologic $\mathrm{pH}$ range ( $\mathrm{pH} 1.2, \mathrm{pH} 4.5, \mathrm{pH}$ 6.8). A model-independent approach of difference $(f 1)$ and similarity ( $f 2)$ tests were applied to dissolution data. A clinical study was performed with 16 patients who were divided into two groups - one group received formulation $A(n=9)$ and the other received formulation $B(n=7)$. Lamotrigine steady-state concentrations were determined by high-performance liquid chromatography on a reverse-phase column.

Results: There were no statistically significant differences in lamotrigine serum concentrations between the two groups, although formulation B had slightly higher mean concentration values (formulation A: $3.97 \pm 4.1 \mu \mathrm{g} / \mathrm{mL}$; formulation B: $5.78 \pm 2.7 \mu \mathrm{g} / \mathrm{mL}$ ). Dissolution profiles of the two formulations were similar in the $\mathrm{pH} 1.2$ dissolution medium; however, the dissolution profiles of formulation $\mathrm{B}$ were outside the dissolution limit $(\geq 85 \%$ at 15 minutes) in the $\mathrm{pH} 4.5$ and 6.8 dissolution media.

Conclusions: No significant changes in the serum concentrations of lamotrigine were seen between the two investigated formulations. There is no 
evidence to suggest that the differences in dissolution profiles at $\mathrm{pH} 4.5$ and $\mathrm{pH} 6.8$ affect the therapeutic efficacy of the formulations. It is evident that the doses of test formulation given to the patients were higher as a consequence of common assumption that generic products have a lower absorption rate, which is proven unnecessary in this study. This investigation was a pilot study and thus further investigations with a larger sample size are necessary to determine if there is a connection between dissolution profiles and the therapeutic effect of investigated formulations.

\section{Introduction}

Epilepsy is a chronic disease that often requires life-long antiepileptic drug (AED) treatment. The primary goal of AED treatment is the avoidance of seizures with no or minimal adverse effects. ${ }^{[1]}$ When long-term seizure remission has been achieved, even a single breakthrough seizure can have serious clinical and social consequences. ${ }^{[2-8]}$ Healthcare systems tend to favor the use of generic drugs in order to reduce medical costs, despite a number of potential disadvantages of generic drugs. ${ }^{[2,9]}$ In the treatment of epilepsy, generic formulations of AEDs are widely used. Although generic substitution of AEDs may be appropriate for many patients, there appear to be a substantial number of patients in whom generic substitution may represent a suboptimal level of care. Issues related to AEDs that should be taken into consideration include their insolubility in water, narrow therapeutic index, non-linear pharmacokinetic profiles, and potential for adverse events. However, breakthrough seizures or adverse reactions due to generic substitution have been reported for AEDs with linear pharmacokinetic profiles and wide therapeutic ranges, such as lamotrigine. ${ }^{[9,10]}$

After the expiration of the patent on the original product $\left(\right.$ Lamictal ${ }^{\circledR}$ ) on 1 June 2005 , it became possible in Serbia to market generic products containing lamotrigine. The linear pharmacokinetics and wide therapeutic window of lamotrigine ${ }^{[11]}$ enable seizure-free patients to easily switch from brand to generic AEDs.

Lamotrigine is in class II of the biopharmaceutical system of classification (BSC). After oral administration, lamotrigine is rapidly ab- sorbed with an absolute bioavailability of $98 \% .^{[12-14]}$ Lamotrigine is a weak base (dissociation constant $\mathrm{pKa}=5.5)$, is poorly soluble in water $(0.17 \mathrm{mg} / \mathrm{mL}$ at $25^{\circ} \mathrm{C}$ ), with a $\log$ octanol/water partition coefficient $(\log \mathrm{P})$ of 1.19 at $\mathrm{pH}$ 7.6. ${ }^{[15]}$ Absorption of lamotrigine after oral tablet administration depends on the release of the drug substance from the drug product, and hence, the dissolution or solubility of the drug under physiologic conditions. Because of the critical nature of these steps, in vitro dissolution may be relevant to the prediction of in vivo performance. Generic tablets of lamotrigine contain different substances to branded tablets, which may affect the release and pharmacokinetics of the active substance. Big differences between lamotrigine preparations could cause relapses in seizure-free patients or toxic reactions.

Although studies of bioequivalence in different lamotrigine tablet formulations are conducted, they are performed in healthy subjects and not in patients. The patient population is likely to have greater variability in terms of characteristics. ${ }^{[16]}$ For example, patients with epilepsy are likely to span a larger age range than the adults used in standard tests, which may result in differences in drug-handling characteristics. ${ }^{[17]}$ In addition, only single-dose studies are performed, which are not relevant to the continued use of AEDs. If one product is bioequivalent to another, that does not guarantee that a drug will have the same therapeutic and adverse effect profile as the reference drug. ${ }^{[2,18]}$ It is not yet known whether absorption rates between different lamotrigine tablet formulations are the same. Some studies have shown that differences in absorption rates correlate with dose-dependent adverse effects. ${ }^{[19,20]}$ 
In this study, the extent of variations in lamotrigine serum concentrations between two immediate-release tablet formulations was investigated. Data were correlated with in vitro difference and similarity tests on dissolution profiles of the two formulations.

\section{Materials and Methods}

\section{Clinical Study}

This study was approved by the Medical Faculty Ethics Committee at the University of Novi Sad, Novi Sad, Republic of Serbia. Written informed consent was obtained from all patients or their guardians. Demographic data were collected from all patients. Enrollment criteria included epileptic patients who were receiving lamotrigine monotherapy (i.e. formulation A: Lamictal ${ }^{\circledR}$ $100 \mathrm{mg}$ tablets [GlaxoSmithKline, UK, Lot: 062656, expiry date: 07.2010]; or formulation B: Lamal $^{\circledR} 100 \mathrm{mg}$ tablets [Alkaloid, Skoplje, Macedonia, Lot: 07593 0308, expiry date: 03.2011]) and had not missed a dose for 3 weeks prior to enrollment (i.e. only patients with steady-state lamotrigine were included). Individual lamotrigine doses that were optimal for seizure control ranged from 50 to $300 \mathrm{mg} / \mathrm{day}$, and were taken 30 minutes before breakfast and 30 minutes before the evening meal as instructed by investigators. Seizure control and dose-related CNS adverse effects (e.g. dizziness, ataxia, diplopia, blurred vision, nausea, and vomiting) were investigated in all patients. Patients were divided into two main groups. The first group consisted of patients taking formulation A $(n=9)$, while the second group consisted of patients taking formulation $\mathrm{B}$ $(n=7)$. All patients were initially receiving formulation A, before the seven patients were switched to formulation B for cost-saving reasons; treatment with formulation A cost $€ 4$, but formulation B cost only 20c because it was subsidized by the healthcare system. Patients were treated for at least 6 months with formulation A or formulation $\mathrm{B}$ before the pharmacokinetic study. Both formulations were taken twice a day. During the study, blood samples were taken from each patient once a day. Patients were asked to report precisely the time of drug administration, and blood samples were collected accordingly. The time between drug administration and sample collection was between 1.5 and 8 hours. All statistical calculations were performed using SPSS $^{\circledR}$ version 17 (Chicago, IL, USA). Statistical significance was tested by Student's t-test. A p-value of $<0.05$ was considered significant.

\section{Dissolution Testing and Sample Preparation}

In vitro dissolution of formulations $\mathrm{A}$ and B was performed according to the published method. ${ }^{[21]}$ The dissolution test was performed by using Erweka dissolution apparatus (Erweka, DT800, Germany). Three dissolution media $(900 \mathrm{~mL})$ were used over the physiologic $\mathrm{pH}$ range of $1.2-6.8(\mathrm{pH} 1.2 \mathrm{HCl}$ solution, $\mathrm{pH} 4.5$ acetate buffer, $\mathrm{pH} 6.8$ phosphate buffer), with six tablets being analyzed for each medium. The paddle assembly was used (USP apparatus 2), revolution speed was kept at $50 \mathrm{rpm}$. In all experiments, $5 \mathrm{~mL}$ of dissolution sample was withdrawn at $5,10,15,30,45$, and 60 minutes. $5 \mathrm{~mL}$ of fresh medium were replaced after sampling. Experiments were conducted in triplicate (i.e. a total of 18 tablets tested per medium). Samples were filtered through a $0.45 \mu \mathrm{m}$ porosity filter. $100 \mu \mathrm{L}$ of the filtered solution was diluted with $900 \mu \mathrm{L}$ of mobile phase solution used for highperformance liquid chromatography (HPLC) analysis. $20 \mu \mathrm{L}$ of each sample was then injected into the HPLC system.

\section{Chromatographic Conditions for Dissolution Samples}

Chromatographic separation was performed on an HPLC system (Agilent Technology Series 1100 , USA), which consisted of an autoinjector, a C18 guard column $(4.6 \times 12.5 \mathrm{~mm}, 5 \mu \mathrm{m}$, Zorbax, Agilent Technology, USA), a C18 analytical column $(4.6 \times 250 \mathrm{~mm}, 5 \mu \mathrm{m}$, Zorbax, Agilent Technology, USA), and a diode-array detector (DAD) $[210 \mathrm{~nm}]$. The mobile phase solution was a mixture of phosphate buffer $(0.05 \mathrm{~mol} / \mathrm{L}$, adjusted to $\mathrm{pH} 2.68$ with $40 \%$ ortho-phosphoric acid) and acetonitrile $(68: 32, \mathrm{v} / \mathrm{v})$. All separations were performed isocratically at a flow rate of $1.2 \mathrm{~mL} / \mathrm{min}$. 
Table I. Dissolution tests of formulation A (original lamotrigine formulation) and formulation B (generic lamotrigine formulation) in three different $\mathrm{pH}$ media $(\mathrm{pH} 1.2 \mathrm{HCl}$ solution, $\mathrm{pH} 4.5$ acetate buffer, and $\mathrm{pH} 6.8$ phosphate buffer)

\begin{tabular}{|c|c|c|c|c|c|c|}
\hline \multirow[t]{3}{*}{$\overline{T i m e}(\min )$} & \multicolumn{6}{|c|}{ Dissolution (\%) [ \pm SD] } \\
\hline & \multicolumn{2}{|l|}{$\overline{\mathrm{pH}} 1.2$} & \multicolumn{2}{|l|}{$\mathrm{pH} 4.5$} & \multicolumn{2}{|l|}{$\mathrm{pH} 6.8$} \\
\hline & $\bar{A}$ & B & $\bar{A}$ & B & $\bar{A}$ & $B$ \\
\hline 5 & $95.13 \pm 4.84$ & $90.20 \pm 5.32$ & $87.98 \pm 1.55$ & $70.44 \pm 5.82$ & $69.74 \pm 1.32$ & $59.55 \pm 4.17$ \\
\hline 10 & $99.34 \pm 2.19$ & $95.61 \pm 2.51$ & $87.34 \pm 2.18$ & $75.82 \pm 2.66$ & $83.53 \pm 1.30$ & $62.84 \pm 3.06$ \\
\hline 15 & $100.69 \pm 3.14$ & $97.52 \pm 3.98$ & $96.48 \pm 1.28$ & $79.91 \pm 5.17$ & $95.06 \pm 1.91$ & $65.98 \pm 2.46$ \\
\hline 30 & $100.84 \pm 3.14$ & $98.43 \pm 2.82$ & $98.77 \pm 3.21$ & $80.72 \pm 2.98$ & $97.55 \pm 3.97$ & $67.32 \pm 3.15$ \\
\hline 45 & $100.31 \pm 3.24$ & $100.1 \pm 4.11$ & $98.79 \pm 4.40$ & $85.78 \pm 4.44$ & $99.72 \pm 1.28$ & $71.41 \pm 2.27$ \\
\hline 60 & $100.77 \pm 2.92$ & $100.66 \pm 3.40$ & $99.6 \pm 2.50$ & $86.91 \pm 4.49$ & $100.86 \pm 2.45$ & $71.01 \pm 2.14$ \\
\hline
\end{tabular}

\section{Blood Sample Preparation}

Blood samples were collected in non-heparinized tubes and centrifuged at $3000 \mathrm{rpm}$ for 15 minutes. Serum was separated, transferred into test tubes and stored at $-20^{\circ} \mathrm{C}$ prior to analysis. Before chromatographic analyses, one part of serum was mixed with two parts of acetonitrile, centrifuged at $5000 \mathrm{rpm}$ for 10 minutes. The supernatant $(10 \mu \mathrm{L})$ was injected into the HPLC system.

\section{Chromatographic Conditions for Serum Samples}

Serum samples were analyzed for lamotrigine concentrations at steady state using a published method and the same HPLC system as for dissolution samples. ${ }^{[22]}$ The mobile phase solution was a mixture of potassium dihydrogen phosphate buffer $(10 \mathrm{mmol} / \mathrm{L}$, adjusted to $\mathrm{pH} 7$ using $0.5 \mathrm{~mol} / \mathrm{L} \mathrm{NaOH})$, methanol, acetonitrile, and acetone $(55: 22: 12: 11 \mathrm{v} / \mathrm{v})$. The flow rate was $1.2 \mathrm{~mL} / \mathrm{min}$. Under these conditions, retention time for lamotrigine was 4.3 minutes. The calibration curve was linear over the concentration range of $0.5-40 \mu \mathrm{g} / \mathrm{mL}$ of lamotrigine, with a coefficient of determination $\left(r^{2}\right)$ of $\geq 0.989$.

\section{Results}

\section{In Vitro study}

In vitro dissolution of formulations $\mathrm{A}$ and $\mathrm{B}$ was performed to obtain information about possible differences between their dissolution profiles and, consequently, differences in bioavailability.
Cumulative percentages of the drug dissolved from the tablets were calculated over experimentally obtained drug content. Results of the dissolution tests are shown in table I, and dissolution profile curves in figures 1 and 2 .

The fastest dissolution rate of drug was obtained at $\mathrm{pH} 1.2$ for both formulations because lamotrigine has the highest solubility in this medium. The $\mathrm{pH}$ value of the medium had a marked effect on the dissolution profile of formulation B, while formulation A had similar profiles in all three media, which is in accordance with previously reported results. ${ }^{[21]}$ The dissolution limit that was set at $\geq 85 \%$ at 15 minutes by the USP (XXVIII) was not fulfilled for formulation $\mathrm{B}$ in $\mathrm{pH} 4.5$ and $\mathrm{pH} 6.8$ media (figure 2, table I). ${ }^{[23]}$

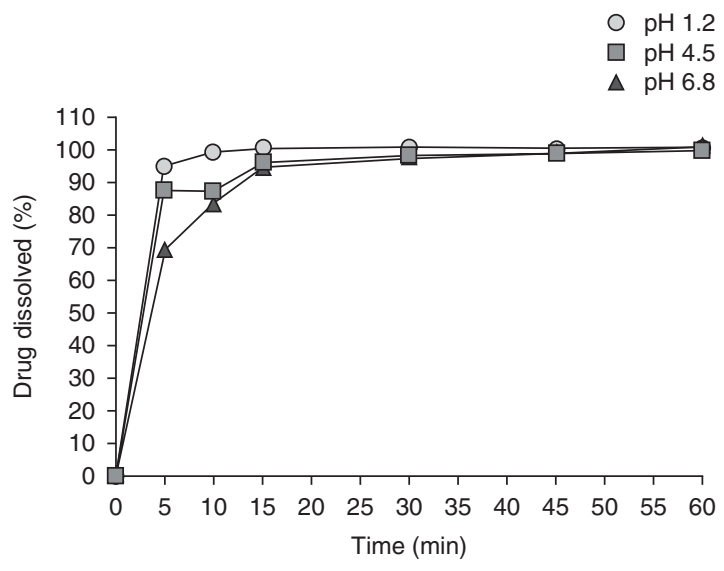

Fig. 1. Dissolution profile of formulation $A$ (original formulation) in three different $\mathrm{pH}$ media $(\mathrm{pH} 1.2 \mathrm{HCl} ; \mathrm{pH} 4.5$ acetate buffer; $\mathrm{pH} 6.8$ phosphate buffer). 


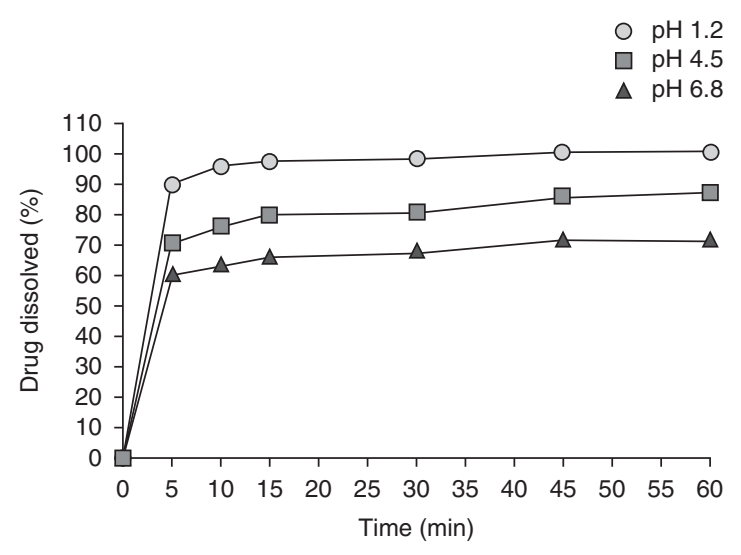

Fig. 2. Dissolution profile of formulation $B$ (generic formulation) in three different $\mathrm{pH}$ media $(\mathrm{pH} 1.2 \mathrm{HCl} ; \mathrm{pH} 4.5$ acetate buffer; $\mathrm{pH} 6.8$ phosphate buffer).

\section{Difference and Similarity Test}

A model-independent approach of difference $(f 1)$ and similarity $(f 2)$ tests were applied to dissolution data. The values of $f 1$ and $f 2$ factors for the test product (formulation $\mathrm{B}$ ) versus the reference (formulation A) were calculated from the means of the percentage of drug dissolved at each timepoint (table I) by using equations 1 and 2. ${ }^{[24]}$

$$
\begin{gathered}
f_{1}=\left\{\frac{\left[\sum_{t=1}^{n}\left|R_{t}-T_{t}\right|\right]}{\left[\sum_{t} \frac{R_{t}+T_{t}}{2}\right]}\right\} \times 100 \\
f_{2}=50 \times \\
\log \left\{\left[1+(1 / n) \sum_{t=1}^{n}\left(R_{t}-T_{t}\right)^{2}\right]^{-0.5} \times 100\right\}
\end{gathered}
$$

where $n$ is the number of dissolution sample times, and $R_{t}$ and $T_{t}$ are the individual or mean percentage of drug dissolved at each timepoint $(t)$, for the reference and test dissolution profiles, respectively. According to the US FDA guidance, $f 1$ values generally vary between 0 and 15 , and $f 2$ values vary between 50 and 100 , ensuring equivalence of the dissolution curves. ${ }^{[25]}$ Dissolution profiles of formulations A and B were only similar in the $\mathrm{pH} 1.2$ medium (table II).

\section{Clinical Study}

The dissolution data were compared with clinical data of randomly chosen patients receiv- ing monotherapy with formulation $\mathrm{A}(\mathrm{n}=9[\mathrm{n}=5$ females, $n=4$ males] $)$ or formulation $B(n=7$ $[\mathrm{n}=3$ females, $\mathrm{n}=4$ males]). Dosage details and patient characteristic are shown in table III.

There was a statistically significant difference between serum concentrations of lamotrigine in patients receiving formulation A $(3.14 \pm 1.95 \mu \mathrm{g} / \mathrm{mL})$ compared with formulation B $(5.78 \pm 2.78 \mu \mathrm{g} / \mathrm{mL})$ [figure 3a]. The range of lamotrigine serum concentrations was between 1.03 and $7.44 \mu \mathrm{g} / \mathrm{mL}$ for formulation A, and between 1.03 and $9.44 \mu \mathrm{g} / \mathrm{mL}$ for formulation B. Most patients receiving formulation A had plasma concentrations of lamotrigine $<3 \mu \mathrm{g} / \mathrm{mL}$ (five of nine). However, the majority of lamotrigine plasma concentrations (six of seven) in patients receiving formulation $\mathrm{B}$ were in the range of 3 to $14 \mu \mathrm{g} / \mathrm{mL}$, which has previously been suggested as the therapeutic range. ${ }^{[26,27]}$ No dose-dependent adverse effects appeared in the patients, and all patients were seizure free. No statistically significant differences were found between dose-normalized concentrations of these two formulations (figure $3 b$ ).

The relationship between lamotrigine serum concentration $(\mu \mathrm{g} / \mathrm{mL})$ and lamotrigine dose $(\mathrm{mg} / \mathrm{kg} /$ day $)$ were linear in both formulations $\left(r^{2}=0.78484\right.$ formulation $\mathrm{A} ; \mathrm{r}^{2}=0.83417$ formulation B) [figure 4]. Formulation A had a lower standard deviation (SD) and data scattering because, as the dissolution data showed, it is less influenced by $\mathrm{pH}$ changes. The test formulation, or formulation $\mathrm{B}$, had a higher data scattering and $\mathrm{SD}$, which corresponds with the higher influence of $\mathrm{pH}$ changes on the dissolution profile.

\section{Discussion}

This study showed that the investigated lamotrigine tablet formulations have different dissolution

Table II. Difference $(f 1)$ and similarity $(f 2)$ factors for reference (formulation A - original formulation) vs test product (formulation B generic formulation)

\begin{tabular}{lcl}
\hline Dissolution medium & Difference factor $(f 1)$ & Similarity factor $(f 2)$ \\
\hline $\mathrm{pH} \mathrm{1.2} \mathrm{HCl} \mathrm{solution}$ & 2.46 & 74.99 \\
$\mathrm{pH} 4.5$ acetate buffer & 17.05 & 40.86 \\
$\mathrm{pH} \mathrm{6.8} \mathrm{phosphate}$ & 31.41 & 29.43 \\
buffer & & \\
\hline
\end{tabular}


Table III. Dosing details of lamotrigine and summary of patients characteristics for formulation A (original formulation) and formulation B (generic formulation) $^{\mathrm{a}}$

\begin{tabular}{lll}
\hline & Formulation A & Formulation B \\
\hline No. of patients & 9 & 7 \\
Dosing details & & \\
$\quad$ dose of lamotrigine $(\mathrm{mg} / \mathrm{kg} /$ day $)$ & $2.02 \pm 0.85^{*}$ & $2.94 \pm 1.52^{*}$ \\
$\quad$ lamotrigine serum concentration $(\mu \mathrm{g} / \mathrm{mL})$ & $3.14 \pm 1.95^{*}$ & $5.78 \pm 2.78^{*}$ \\
$\quad$ lamotrigine dose-normalized concentration & $1.68 \pm 0.99^{*}$ & $2.35 \pm 2.04^{*}$ \\
Characteristics & & $25.86 \pm 12.88$ \\
$\quad$ age $(\mathrm{y})$ & $27.11 \pm 5.86$ & $172.00 \pm 5.23$ \\
$\quad$ height $(\mathrm{cm})$ & $176.33 \pm 9.91$ & $64.57 \pm 7.52$ \\
$\quad$ weight $(\mathrm{kg})$ & $71.33 \pm 14.92$ & \\
\hline $\mathrm{a} \quad$ Data are given as mean $\pm \mathrm{SD}$. & & \\
$* \mathrm{p}<0.05$. & & \\
\hline
\end{tabular}

profiles because they differ in the manufacturing process used and in the substances with which the active principle is associated. ${ }^{[28]}$ These differences have influences on the dissolution rates in the gastrointestinal tract, as well as absorption, and, hence, the bioavailability of lamotrigine.

Results of the dissolution tests were in correlation with the chemical properties of lamotrigine. As a basic drug, lamotrigine had high solubility only in $\mathrm{pH} 1.2 \mathrm{HCl}$ medium, ${ }^{[29]}$ thus, the dissolution profiles were similar only in acid medium. According to some investigations, there is good correlation on level A between in vitro and in vivo data of lamotrigine. ${ }^{[29]}$ Lamotrigine is in class II of the BSC and its dissolution is the rate-limiting step for absorption and bioavailability of this drug. ${ }^{[29,30]}$ In vitro data indicated that dissolution media had an impact on the dissolution profiles of these two formulations in two of the three different $\mathrm{pH}$ media. Thus, the variations in lamotrigine serum concentrations within patients receiving these products was due to the fact that the main dissolution and absorption should take place in the stomach environment. According to some studies, after ingestion of drug in the fasted state, with about $200 \mathrm{~mL}$ of water, the $\mathrm{pH}$ in the stomach can rise up to 4.5 , and it needs about 60 minutes to return to the equilibrium of $\mathrm{pH}$ 1.2. With ingestion of food (i.e. fed state), the stomach $\mathrm{pH}$ could rise up to 7 , and it takes more than 90 minutes to reach equilibrium. ${ }^{[31]}$ Since the content of the stomach in the fasted state is
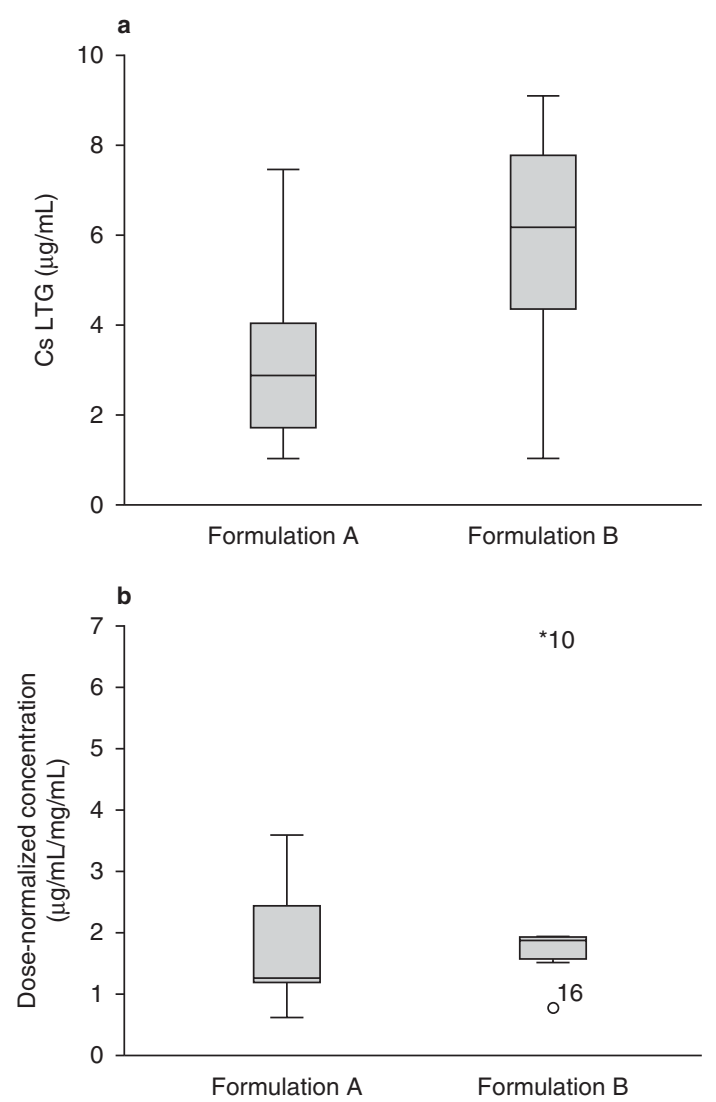

Fig. 3. (a) Lamotrigine (LTG) serum concentrations (Cs); and (b) dose-normalized concentrations of formulations $A$ (original formulation) and B (generic formulation) ['10' represents an extreme case and ' 16 ' is an outlier value]. 


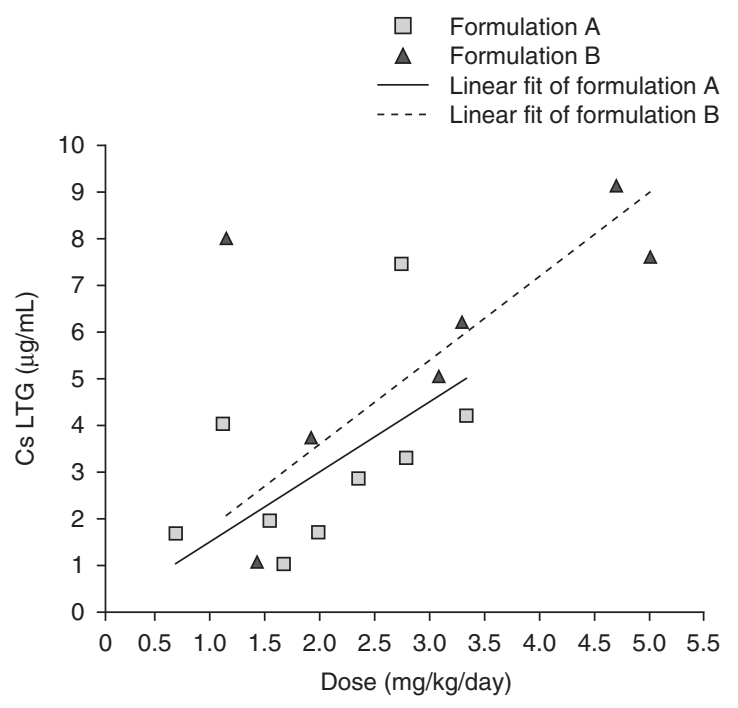

Fig. 4. Lamotrigine (LTG) serum concentrations (Cs) vs LTG daily dose.

emptied every 15 minutes and in the fed state every 30-60 minutes, observed statistical differences in serum concentrations of the two tested formulations is justified.

Patients receiving formulation $\mathrm{B}$ actually received a higher lamotrigine dose than those treated with formulation A, although there were no statistically significant differences between doses.

It is a common assumption that generic drugs have a lower absorption rate and therapeutic efficiency than branded drugs and therefore patients are given higher doses of generic drugs, which is proven unnecessary in this investigation. The variation in plasma concentrations could also be due to individual characteristics of patients (i.e. autoinduction and race), but they are unlikely to be clinically significant. ${ }^{[32]}$ There is no evidence to suggest that concentration variations of lamotrigine were caused by the differences in formulations.

Although switching of phenytoin, carabamazepine, and valproate is restricted in many countries, switching between lamotrigine products in most European countries is permitted. However, in some countries, such as Sweden, it is specified that lamotrigine products cannot be replaced with one another, since breakthrough seizures or adverse reactions due to substitution have been reported. ${ }^{[9,10,33]}$ This pilot study supports evidence for the ability to switch between lamotrigine immediate-release tablet products with no consequences on the health of the patient.

\section{Conclusions}

Although dissolution profiles of the two formulations are only similar in the $\mathrm{pH} 1.2$ medium, switching between formulations A and B did not cause any dose-dependent adverse events and worsening of seizure control. Taking into consideration that five of nine patients receiving formulation A had serum concentrations of lamotrigine that were under the proposed therapeutic range, and this was only one in seven in formulation B, there is no evidence to support the fact that therapeutic efficacy of formulation $\mathrm{B}$ is lower because its dissolution profile was out of specification in $\mathrm{pH} 4.5$ and $\mathrm{pH} 6.8$ media. This pilot study supports evidence for the ability to switch between these two lamotrigine tablet formulations.

\section{Acknowledgments}

This study has been supported by project no. 145060 of the Ministry of Science and Technology, Republic of Serbia. The authors have declared that they have no conflicts of interest that are directly relevant to the content of this article.

\section{References}

1. EUCARE. European white paper in epilepsy. Epilepsia 2003; 44 (6): 1-88

2. Besag FMC. Is generic prescribing acceptable in epilepsy? Drug Safety 2000; 23: 173-82

3. Kramer G, Schneble H, Wolf P. Risks of the new "aut idem" regulations for treatment with antiepileptic drugs. Akt Neurol 2002; 29: 115-22

4. Jacoby A, Baker G, Steen N, et al. The clinical course of epilepsy and its psychosocial correlate: findings from a UK community study. Epilepsia 1996; 37: 148-61

5. Crawford P, Hall WW, Chappell B, et al. Generic prescribing for epilepsy: is it safe? Seizure 1996; 5: 1-5

6. Anonymous. For and against generic prescribing. Drug Ther Bull 1987; 25: 93-5

7. Meyer MC, Straughn AB. Biopharamaceutical factors in seizure control and drug toxicity. Am J Hosp Pharm 1993; 50 (5): S17-22

8. Andermann F, Duh MS, Gosselin A, et al. Compulsory generic switches of antriepileptic drugs: high switchback rates 
to branded compounds compared with other drug classes. Epilepsia 2007; 48: 464-9

9. Blier P. Brand versus generic medications: the money, the patients and the research. J Psychiatry Neurosci 2003; 28: $167-8$

10. Markus GM, McCormick J. Identification of adverse reactions that can occur on substitution of generic for branded lamotrigine in patients with epilepsy. Clin Ther 2007; 29: 1249-50

11. Cohen AF, Land GS, Breimer DD, et al. Lamotrigine, a new anticonvulsant: pharmacokinetics in normal humans. Clin Pharmacol Ther 1987; 42: 535-41

12. Levy RH, Mattson RH, Meldrum BS, et al. Antiepileptic drugs. 5th rev. ed. Philadelphia (PA): Lippincott Williams and Wilkins, 2002

13. Physicians' Desk Reference. Toronto (ON): Thomson PDR, 2007; 61: 1481-90

14. Sweetman S. Martindale: the complete drug reference London: Pharmaceutical Press, 2007

15. Thaddeus HG, Fiedler-Kelly J, Cox E, et al. Population pharmacokinetics of lamotrigine adjunctive therapy in adults with epilepsy. J Clin Pharmacol 1999; 39: 373-84

16. Bialer M. Generic products of antiepileptic drugs (AEDs): is it an issue? Epilepsia 2007; 48 (10): 1825-32

17. Meredith PA. Generic drugs: therapeutic equivalence. Drug Saf 1996; 15: 233-42

18. Wilner AN. Therapeutic equivalency of generic antiepileptic drugs: results of a survey. Epilepsy Behav 2004; 5: 995-8

19. Kramer G, Biraen A, Carreno M, et al. Current approaches to the use of generic antiepileptic drugs. Epilpsy Behav 2007; 11: 46-52

20. Nielsen KA, Dahl M, Tommerup E, et al. Comparative daily profile with different preparations of lamotrigine: a pilot investigation. Epilepsy Behav 2008; 13: 127-30

21. Sripalakit P, Srichaiya A, Kandee R. Development and validation of a HPLC method for a dissolution test of lamotrigine tablets and its application to drug quality control studies. J Liquid Chromatography \& Related Technologies 2008; 31: 926-39

22. Patil KM, Bodhankar SL. Simultaneous determination of lamotrigine, phenobarbitone, carbamazepine and phenytoin in human serum by high-performance liquid chromatography. J Pharmaceut Biomed 2005; 39: 181-6
23. United States Pharmacopoeial Convention. The United States Pharmacopoeia. 28th ed. Rockville (MD) 2005

24. Paul C, Sousa Lobo JM. Modeling and comparation of dissolution profiles. Eur J Pharm Sci 2001; 13: 123-33

25. Guidance for Industry. Dissolution testing of immediate release solid oral dosage forms. Rockville (MD): U.S. Department of Health and Human Services, Food and Drug Administration, Center for Drug Evaluation and Research, 1997 Aug

26. Morris RG, Black AB, Harris AL, et al. Lamotrigine and therapeutic drug monitoring: retrospective survey following the introduction of routine service. Br Clin Pharmacol 1998; 46: 547-51

27. Lalic M, Cvejic J, Popovic J, et al. Lamotrigine and valproate pharmacokinetics interactions in epileptic patients. Eur J Drug Metab Pharmacokinet 2009; 34 (2): 93-9

28. Guberman A, Corman C. Generic substitution for brand name antiepileptic drugs: a survey. Can J Neurol Sci 2000; 27: $37-43$

29. Souliman S, Blanquet S, Beyssac E, et al. A level A in vitro/ in vivo correlation in fasted and fed states using different methods: applied to solid immediate release oral dosage form. Eur J Pharm Sci 2006; 27: 72-9

30. Lue BM, Nielsen FS, Magnussen T, et al. Using biorelevant dissolution to obtain IVIC of solid dosage forms containing a poorly-soluble model compound. Eur J Pharm Biopharm 2008; 69: 648-57

31. Shah HJ, Subbaiah G, Patel DM, et al. In vitro-in vivo correlation of modified release dosage form of lamotrigine. Biopharm Drug Dispos 2009; 30: 524-31

32. Hussein Z, Posner J. Population pharmacokinetics of lamotrigine monotherapy in patients with epilepsy: retrospective analysis of routine monitoring data. $\mathrm{Br} \mathbf{J}$ Clin Pharmacol 1997; 43: 457-65

33. MPA (Läkemedelsverket) substitutable medical products. Medical Products Agency. Uppsala: Läkemedelsverket, 2006

Correspondence: Dr Jelena Cvejic, Medical Faculty, Department of Pharmacy, University of Novi Sad, Hajduk Veljkova 3, 21000 Novi Sad, Republic of Serbia.

E-mail: cvejich@hotmail.com 